\title{
Rigidisation of Deployable Space Polymer Membranes by Heat-Activated Self-Folding
}

DOI:

10.1088/1361-665X/aadc72

\section{Document Version}

Accepted author manuscript

Link to publication record in Manchester Research Explorer

\section{Citation for published version (APA):}

Wu, R., Roberts, P., Lyu, S., Soutis, C., Zheng, F., Diver, C., Gresil, M., \& Blaker, J. (2018). Rigidisation of Deployable Space Polymer Membranes by Heat-Activated Self-Folding. Smart Materials \& Structures, 27, [105037]. https://doi.org/10.1088/1361-665X/aadc72

\section{Published in:}

Smart Materials \& Structures

\section{Citing this paper}

Please note that where the full-text provided on Manchester Research Explorer is the Author Accepted Manuscript or Proof version this may differ from the final Published version. If citing, it is advised that you check and use the publisher's definitive version.

\section{General rights}

Copyright and moral rights for the publications made accessible in the Research Explorer are retained by the authors and/or other copyright owners and it is a condition of accessing publications that users recognise and abide by the legal requirements associated with these rights.

\section{Takedown policy}

If you believe that this document breaches copyright please refer to the University of Manchester's Takedown Procedures [http://man.ac.uk/04Y6Bo] or contact uml.scholarlycommunications@manchester.ac.uk providing relevant details, so we can investigate your claim.

\section{OPEN ACCESS}




\title{
Rigidisation of Deployable Space Polymer Membranes by Heat-Activated Self-Folding
}

\author{
Rui $\mathbf{W u}^{1}$, Peter C.E. Roberts ${ }^{1}$, Shida Lyu ${ }^{2}$, Constantinos \\ Soutis $^{2}$, Fei Zheng ${ }^{3}$, Carl Diver ${ }^{1}$, Matthieu Gresil ${ }^{2,4}$ and \\ Jonny J Blaker ${ }^{3}$ \\ ${ }^{1}$ School of Mechanical, Aerospace and Civil Engineering, the University of \\ Manchester, UK \\ 2 The University of Manchester Aerospace Research Institute, UK \\ ${ }^{3}$ School of Materials, the University of Manchester, UK \\ 4 i-Composites Lab, School of Materials, The University of Manchester \\ E-mail: rui.wu@manchester.ac.uk
}

\begin{abstract}
Current gossamer space structures such as solar sails usually rely on bracing structures, inflation gas, or centrifugal force to deploy and maintain a structural shape, which leads to a system that is sometimes complicated, while a concise system can be achieved if the gossamer structure could self-rigidise and support load. The present study proposes a self-folding polymer membrane based on space-qualified materials and is potentially mass-producible by industrial rollto-roll processes. It can permanently transform a flat gossamer membrane into a load-bearing 3D configuration when heated by sunlight in space, while the foldinginduced shape bifurcation and buckling are prevented using a kirigami hinge design. The shape transformation is demonstrated in lab by a tubular and an origami structure that are formed from a flat membrane when heated to $82^{\circ} \mathrm{C}$ in oven. Thermal radiation analyses have also verified the feasibility of sunlight-activated folding in space when vapour-deposited metallic coatings are applied onto the hinges. The proposed material offers a new generation of gossamer space membrane that can automatically morph from a stowed configuration to a load-bearing structure, and potentially provide built-in functionalities.
\end{abstract}

Keywords: active material, smart material, morphing, bi-stable, meta-structure, kirigami 


\section{Nomenclatures}

$D$ : flexural rigidity

$E$ : Young's modulus

$I$ : second moment of area

$Q$ : heat transfer

$q_{s}:$ solar constant $=1.36 \mathrm{~kW} / \mathrm{m}^{2}$

$T$ : temperature

$\alpha$ : surface absorptivity

$\epsilon$ : surface emissivity

$\sigma$ : Stefan-Boltzmann constant $=5.67 \times 10^{-8} \mathrm{~W} \cdot \mathrm{m}^{-2} \cdot \mathrm{K}^{-4}$

\section{Introduction}

Various space missions require the deployment of gossamer structures, or in other words, large structures with high flexibility and ultra-low-mass [1, 2], such as the reflective membrane of solar sails [3, 4], drag sails [5], and reflectors for communication antennae or space telescopes [6, 7]. Due to the very limited flexural rigidity of thin membrane materials, their in-space deployment is usually achieved using rigidisable deployable booms that drive the unfolding and constrain the out-ofplane deflection during deployment, whilst achieving load-carrying capability when fully deployed [8]. Efforts have also been made to let the membrane deploy and support itself without bracing structures: the fibrous material called microtruss fabric can elastically return to a flat shape when unrolled from a mandrel, while having low surface density owing to its porosity [9]; inflatable structures utilise an inflation gas to carry compressive structural load and a bladder made of membrane material to carry tensile load [10, 11]. Alternatively, centrifugal force has been proposed to deploy and stiffen gossamer structures, including antennae and solar sails [12, 13, 14]. Such a centrifugal deployment removes the bracing structures and therefore significantly reduces the structural mass and improves packing ratio, though the structural dynamic behaviours can be complicated and problematic [15, 16. Furthermore, it has also been reported that a self-rigidisable membrane material could assist the structural performance of those deployable structures: inflatables could benefit from self-rigidising composite bladders that allows the structure to 
stay rigid after inflation without maintaining the gas pressure [17, 18, 19]; heliogyro, a helicopter-rotor-like centrifugally deployed solar sail could use reinforcing battens to improve dynamic behaviours [20], and a self-assembling batten structure, which utilises a sheet material that automatically rolls up into a slender tube due to residual stress, has been proposed [21].

On the other hand, self-folding sheets based on smart materials which fold up along pre-defined hinges under external stimulus have attracted great interests from researchers during the last decade, as it provides a method to convert a $2 \mathrm{D}$ sheet into a 3D device. Such a device not only achieves load-carrying capacity, but also could provide highly design-able behaviours and serve as a building block of future meta-structures [22, 23, 24, 25, 26]. If mass-produced using space-grade materials, it could lead to a new generation of deployable gossamer structures with self-rigidising features and potentially more built-in functionalities. As an example, previous study has proposed an origami-inspired meta-structure reflector for heliogyro solar sail, which can be formed by folding a 2D film. Utilising its tuneable optical properties and enhanced structural rigidity, a concise and robust heliogyro system with passive self-regulation can be realised [27].

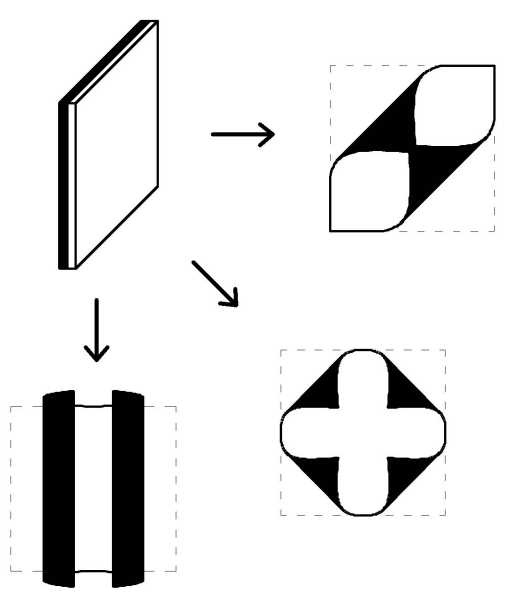

Figure 1: Activation of an isotropic bilayer sheet leads to bifurcation, creating different folded shapes; the white layer is isotropically shrinking.

Self-folding is usually activated using active materials that expand/shrink when stimulated by heat, light, moisture, etc., while the folding (i.e. bending) deformation is achieved by differential expansion/shrinkage through the sheet's thickness. Such 
a differential activation can be realised by either an unsymmetrical stimulus [28] or a double-layer material layup [22, 29, 30, 31]. Meanwhile, the folding induced by an isotropic expansion/shrinkage with a through-the-thickness gradient that is homogeneous across the sheet leads to bifurcation of the final shape (Figure 1), or in other words, the folding can be along any direction [32, 33]. Therefore, a precisely controlled crease pattern requires either an anisotropic active material that expands/shrinks perpendicularly to the hinge line, or an anisotropic flexural rigidity with softened crease lines.

Homogeneous sheet materials with residual tensile stress, which soften and shrink when heated up, can be used to construct self-folding devices (Figure 2a). The temperature gradient leads to different levels of relaxation (thus shrinkage) through the thickness and therefore, activates the folding. Meanwhile, the bifurcation shown in Figure 1 is prevented by localised heating (thus softening) using a laser or lightabsorbing ink [34, 35, 36]. In this case, bifurcation is prevented by anisotropic flexural rigidity. Alternatively, illuminating the sheet material by an ultraviolet light while applying a temporary in-plane unidirectional stretch, then the illuminated side will relax, and a unidirectional compressive stress will build up when the stretching force is removed. When a photo mask is used to constrain the light on certain crease lines, the sheet can be "programmed" to fold into a certain shape [37]. In this case, bifurcation is prevented by anisotropic stress.

Furthermore, various concepts have been proposed utilising a multi-layer architecture. Thin metallic (chromium, copper and nickel) layers bonded onto each other using vapour deposition, lithography and electrodeposition could realise a thin sheet that precisely folds along an origami pattern. The chromium layer with residual stress left by the deposition process actuates the folding of a copper layer, and an anisotropic flexural rigidity is built into the sheet using the reinforcing nickel layer [38, 39, 40]. However, the products are usually at submillimeter size and can not be easily scaled up. Na, Evans et al. [41] have provided an attractive solution (Figure 2b), where a heat shrinking polymer membrane is sandwiched and bonded between two thermally stable polymer membranes, making a trilayer structure. Hinges can be made by cutting off a strip from one of the outer membranes, leaving a bilayer structure at the hinge. The bilayer parts bend when heated up, while the trilayer sections are constrained by the thermally stable membranes thus remains flat. Meanwhile, the removed strip also leads to a region with low flexural rigidity and prevents bifurcation. 


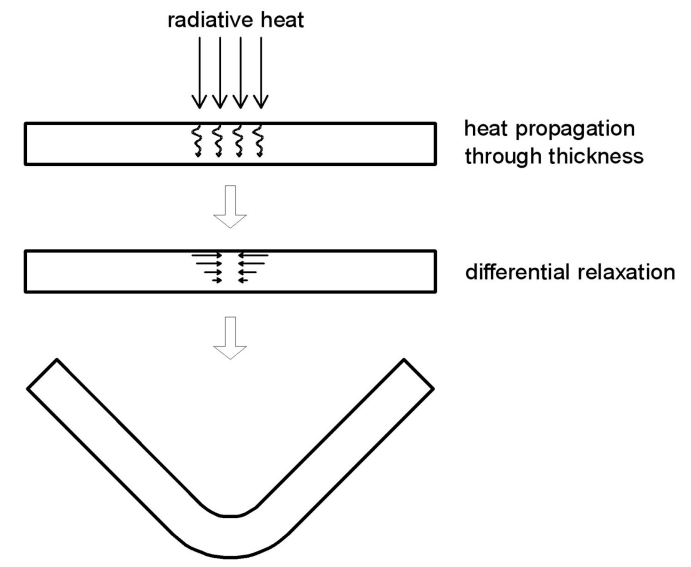

(a)
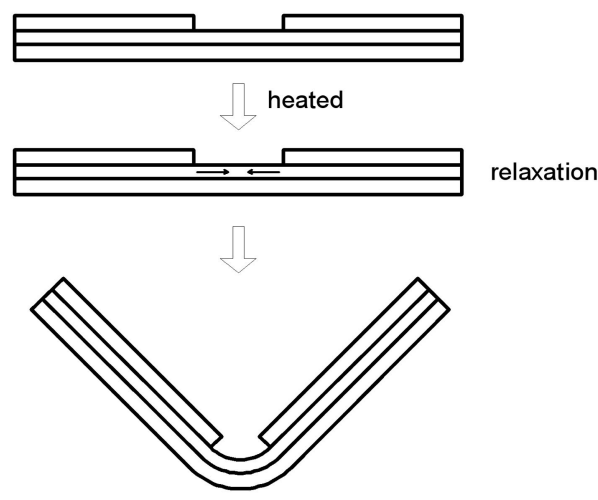

(b)

Figure 2: Two types of existing self-folding membranes, (a) folding induced by differential relaxation through the thickness, (b) folding enabled by multi-layered structure based on designs from [41].

The present study focuses on developing a self-folding thin membrane that can be rolled-up into a cylinder to minimise storage volume, then activated by heat radiation from the sun when unrolled in space. Due to the low sheet thickness and varying lighting conditions, folding by a differential stimulus through its thickness (Figure 2a) is not practical, thus a multi-layered architecture is chosen. The trilayer sheet (Figure 2b) developed by Na, Evans et al. [41] offers a viable solution, but a heavier structure compared to Figure 2a, which makes it unsuitable for certain applications such as solar sails [3].

In the present study, a self-folding Mylar/Kapton composite laminate with kirigami hinges is designed while keeping space application and industrial production in mind.

\section{Design of the self-folding kirigami hinge}

The proposed design uses a thermally stable DuPont ${ }^{\mathrm{TM}}$ Kapton ${ }^{\circledR}$ membrane as the substrate. An isotropic heat shrinking DuPont ${ }^{\mathrm{TM}}$ Mylar ${ }^{\circledR}$ HS film (supplied by UK Insulation Ltd) is the active layer, which is bonded onto the Kapton using $3 \mathrm{M}^{\mathrm{TM}}$ Adhesive Transfer Tape 966. The properties of the three materials are listed in Table 
1 according to manufacturer datasheets. Kapton ${ }^{\circledR}$ is a polyimide, and DuPont ${ }^{\mathrm{TM}}$ Mylar ${ }^{\circledR}$ is made from stretched polyethylene terephthalate (PET), both have been used in space applications. Mylar ${ }^{\circledR}$ HS, where "HS" stands for Heat Shrink, is not heat stabilised and will shrink with considerable force when heated up. The $3 \mathrm{M}^{\mathrm{TM}}$ Adhesive Transfer Tape 966 is made from $3 \mathrm{M}^{\mathrm{TM}}$ High Temperature Acrylic Adhesive 100 , which is a gel-like soft material with low "outgassing" properties.

Table 1: Materials associated with the presented self-folding membrane design.

\begin{tabular}{|c|c|c|c|c|c|c|c|}
\hline Material & $T_{g} /{ }^{\circ} \mathrm{C}$ & Thickness & Modulus/GPa & UT Strength/MPa & UT Elongation & Shrinkage/\% & Operating temp. \\
\hline Kapton $^{(B)} \mathrm{HN}$ & $360 \sim 410$ & $25 \mu m$ & $\begin{array}{l}5\left(-196^{\circ} C\right) \\
2.5\left(23^{\circ} C\right) \\
2\left(200^{\circ} C\right)\end{array}$ & $\begin{array}{c}300\left(-196^{\circ} \mathrm{C}\right) \\
230\left(23^{\circ} \mathrm{C}\right) \\
140\left(200^{\circ} \mathrm{C}\right)\end{array}$ & $\begin{array}{c}19 \%\left(-196^{\circ} \mathrm{C}\right) \\
72 \%\left(23^{\circ} \mathrm{C}\right) \\
83 \%\left(200^{\circ} \mathrm{C}\right)\end{array}$ & $\begin{array}{l}0.17\left(150^{\circ} C\right) \\
1.25\left(400^{\circ} C\right)\end{array}$ & $-269 \sim 400^{\circ} \mathrm{C}$ \\
\hline Mylar ${ }^{(1)}$ HS & 80 & $16 \mu m$ & $3.1\left(23^{\circ} \mathrm{C}\right)$ & $210\left(23^{\circ} \mathrm{C}\right)$ & $115\left(23^{\circ} \mathrm{C}\right) \%$ & $50\left(100^{\circ} \mathrm{C}\right)$ & - \\
\hline Mylar ${ }^{(B)}$ & 80 & $12-38 \mu \mathrm{m}$ & $\begin{array}{c}6.9\left(-70^{\circ} \mathrm{C}\right) \\
3.4\left(23^{\circ} \mathrm{C}\right) \\
0.14\left(200^{\circ} \mathrm{C}\right)\end{array}$ & $\begin{array}{l}200\left(-70^{\circ} \mathrm{C}\right) \\
160\left(23^{\circ} \mathrm{C}\right) \\
70\left(200^{\circ} \mathrm{C}\right)\end{array}$ & $\begin{array}{l}55 \%\left(-70^{\circ} C\right) \\
104 \%\left(23^{\circ} C\right) \\
135 \%\left(100^{\circ} C\right)\end{array}$ & $1.8\left(150^{\circ} \mathrm{C}\right)$ & $-250 \sim 200^{\circ} \mathrm{C}$ \\
\hline 966 acrylic & - & $60 \mu \mathrm{m}$ & gel-like & - & $\gg 100 \%\left(23^{\circ} \mathrm{C}\right)$ & - & $-40 \sim 232^{\circ} \mathrm{C}$ \\
\hline
\end{tabular}

In the present design, Mylar HS is only placed where it is needed: on the kirigami hinges, which guarantees the highly efficient use of the material. Bifurcation is prevented by kirigami structures in the Mylar and adhesive layers that allow the stress along the hinge line to relax, providing a near unidirectional shrinkage that drives the folding. As shown by Figure 3a, a strip of Mylar HS is bonded onto Kapton using the acrylic adhesive. The adhesive is in the form of a transfer tape and therefore has a consistent thickness. Then, through-thickness slits are cut into the Mylar and the adhesive layers using an automatic cutting machine (the detailed process is discussed later in section (3). For the convenience of manufacturing, the slits are not cut throughout the width of the hinge, so that the Mylar strip remains continuous. When heated up, Mylar shrinks and opens the slits (Figure 3b), generating shear strain between Mylar and Kapton, which is accommodated by the flexible adhesive. The opened slits effectively relaxes the shrinkage along $\mathrm{x}$-direction, while the relaxation along y-direction is minor since the Mylar strip's width is higher than (in this case, three times of) the separation between the slits. The membrane thereby folds along the hinge under the y-shrinkage without bifurcation.

However, a tape-spring-type buckling [42] has been observed in test samples (Figure 4a). This is the result of a reversed bending deformation perpendicular to the hinge line triggered by initial unflatness of the membrane, thus will not occur under an ideal condition. Figure 4a also suggests that the reversed bending is facilitated by the slits, which lead to local low bending rigidity and high surface curvature, both 


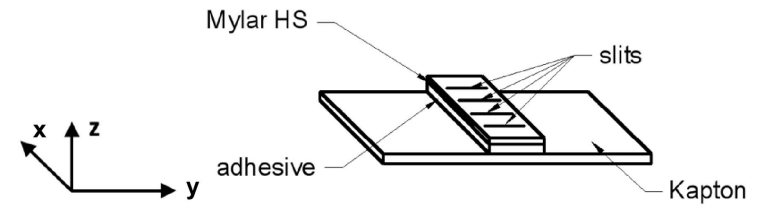

(a)

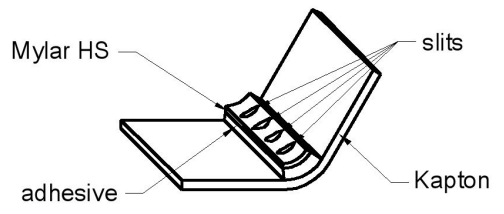

(b)

Figure 3: Schematic draft of the proposed design, containing Kapton substrate and Mylar HS film bonded together through acrylic adhesive, and slits are cut into Mylar and adhesive; (a) a kirigami hinge before activation, (b) a kirigami hinge that after heating, the slits are opened by the shrinking force and the stress along x-direction is relaxed, creating an anisotropic shrinkage that folds the membrane along the hinge

are adverse to the recovery from the buckled shape.

Therefore, the kirigami hinge is modified with staggered slits (design 2 in Figure 4d to prevent a through-width reversed bending and thereby reduce its tendency to buckle. However, fully preventing buckling still requires the membrane to be generally flat when folding is activated, which is discussed in section 5.

The hinge design shown in Figure 4 provides a hinge folding angle close to $180^{\circ}$, which is the maximum folding angle that an origami may require. The folding angle can be seen from Figure $7 \mathrm{~b}$.

\section{Production of the self-folding membrane}

Test samples are fabricated using a partially automatic method. As illustrated by Figure 5b, the production starts with a Mylar HS film bonded to an adhesive transfer tape 966 with a liner adhered to the other side (step 1). Then the layup is loaded onto a CNC (Computer Numerical Control) cutting machine (Figure 5a), which uses a blade to cut the input pattern (hinges profiles and slits) into the Mylar-adhesive layers while leaving the liner mostly intact (step 2). After cutting, the unwanted parts (scrap materials outside the hinge regions, Figure 6b) of the Mylar-adhesive layers are manually removed, leaving only the hinges (step 3). Then the remaining Mylaradhesive layers are transported from the liner onto Kapton using a vinyl transfer tape with low adhesion (step 4-6). Then the above steps are repeated on the other side of the Kapton (step 7). 


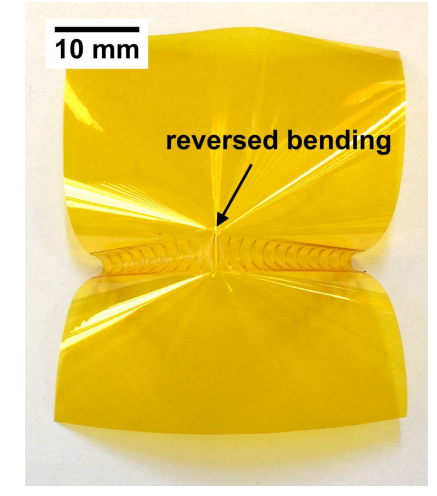

(a)

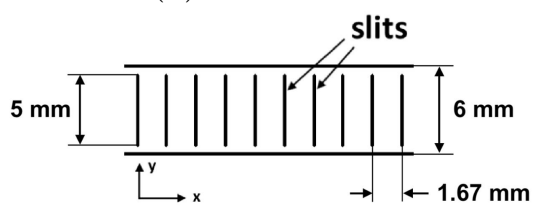

(c)

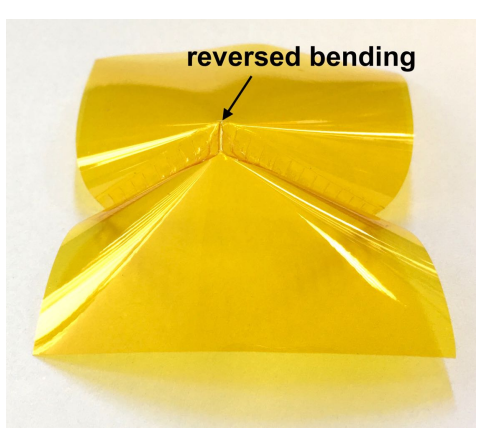

(b)

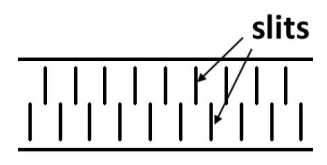

(d)

Figure 4: Design iteration of the kirigami hinge, (a)(b) tape-spring-type buckling of a design 1 kirigami hinge triggered by a reversed bending deformation along a slit, viewed from different angles, (c) design 1 that leads to high tendency of buckling due to the low bending rigidity along a slit, (d) design 2 with staggered slits that offers enhanced flexural rigidity and reduces the tendency of buckling.

This semi-automatic method can be converted to a fully automatic roll-to-roll process. Such a process could enable a cost-effective rapid production of the selffolding membrane and make its large-scale applications feasible. As shown by Figure 6. the process is based on the same steps as in the semi-automatic method (Figure 5). In order to allow the scrap to be easily lifted off from the liner in step 3, the pattern cut into the Mylar and adhesive layer in step 2 can be designed to form one continuous piece of scrap as shown by Figure $6 \mathrm{~b}$.

\section{Folding behaviour of the kirigami hinge}

Folding is activated using an oven where heat is transmitted to the sample by forced air convection at $85^{\circ} \mathrm{C}$, which is slightly higher than the glass transition temperature of Mylar HS $\left(T_{g}=82^{\circ} \mathrm{C}\right)$. It was observed that the hinges without slits failed to fold 


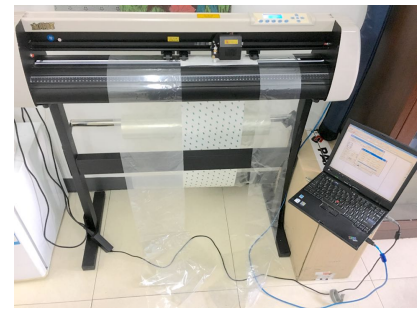

(a)

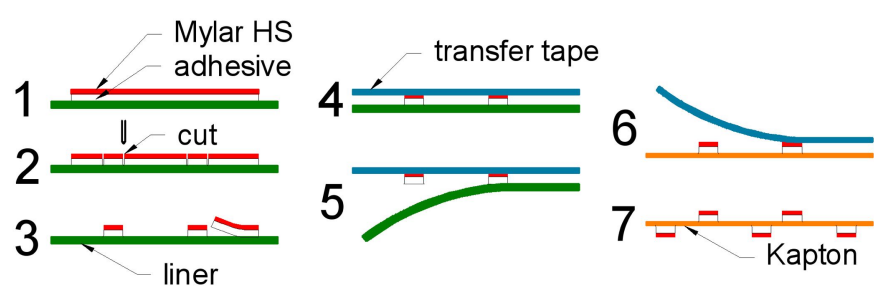

(b)

Figure 5: The semi-automatic method used to fabricate test samples, (a) the CNC pattern cutting machine, (b) the detailed process steps: 1-Mylar+adhesive layer prepared on a liner, 2-pattern cut by the CNC machine, 3-unwanted part removed, leaving only the hinges, 4-transfer tape applied to the top of the Mylar layer, 5-liner removed from the adhesive layer, 6-Mylar-adhesive layer transferred to Kapton, and transfer tape is removed, 7-repeating process 1-6 on the other side of Kapton.

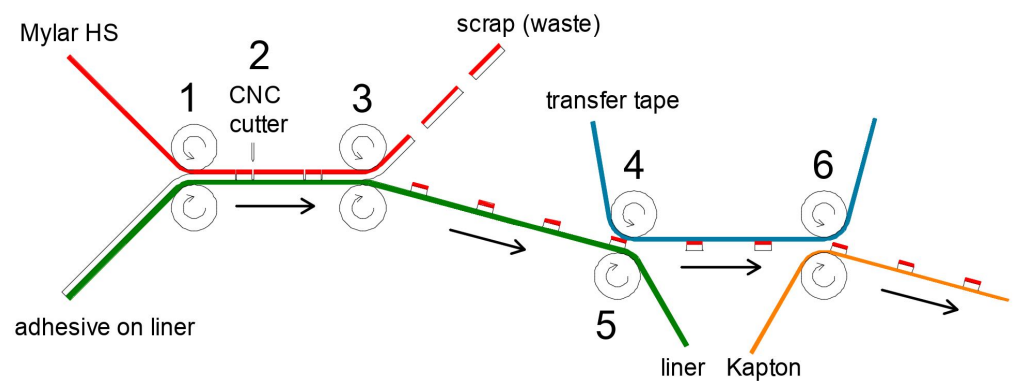

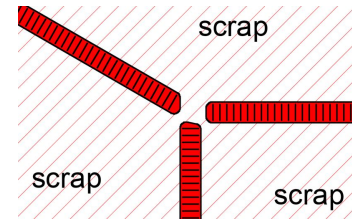

(b)

(a)

Figure 6: The fully automatic roll-to-roll production method converted from the semi-automatic method, (a) the basic process: 1-Mylar rolled onto adhesive on liner, 2-pattern cut by the CNC machine, 3-scrap part removed, leaving only the hinges, 4transfer tape rolled onto the top of the Mylar layer, 5-liner removed from the adhesive layer, 6-Mylar-adhesive layer transferred to Kapton, and transfer tape is removed, then the process $1 \sim 6$ can be repeated on the other side of the Kapton, (b) a typical pattern (thick black lines) cut by the CNC machine, which leaves the scrap in one piece to assist waste removal.

along the hinge line due to bifurcation (Figure 11), while the kirigami hinges folded successfully after $\sim 0.5$ min of heating (Figure 7). 


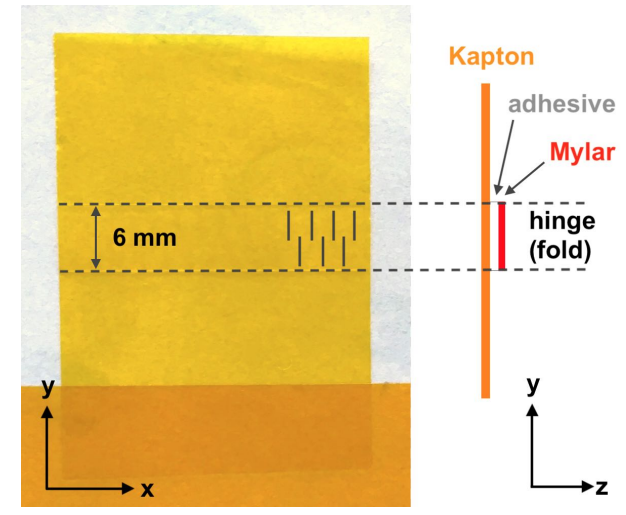

(a)

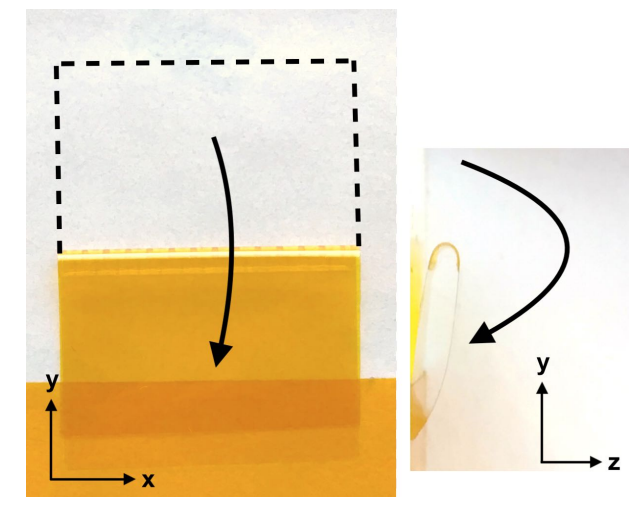

(b)

Figure 7: Test sample with kirigami hinge with hinge design illustrated, (a) before heating, (b) after heating at $85^{\circ} \mathrm{C}$ for $0.5 \mathrm{~min}$, the kirigami hinge successfully folds along the hinge line.

The effect of heating duration is studied by measuring the average shrinkage of the Mylar HS layer at different heating times (Figure 8 and Table 2). Results in Table 2 suggest that the folding is completed after $0.5 \mathrm{~min}$, with no significant shrinkage observed when heating is extended to $6.5 \mathrm{~min}$. The hinge remains in a stable folded configuration after repeated heating and cooling, which is crucial for its space application.

Furthermore, it appears that the shrinkage of Mylar HS has become anisotropic due to relaxation at the densely distributed slits, with more distortion along the $\mathrm{x}$-direction. The $\mathrm{y}$-shrinkage is lower since it is resisted by the Kapton film, and thus induces a higher residual stress after shrinkage. This residual stress enforces a primary folding direction along the hinge line which prevents bifurcation.

Table 2: Anisotropic shrinkage of the Mylar HS layer evaluated from Figure 8 .

\begin{tabular}{c|c|c}
\hline Heating time & Shrinkage along $\mathrm{y}$ & Shrinkage along $\mathrm{x}$ \\
\hline $30 \mathrm{~s}$ & $22 \%$ & $35 \%$ \\
\hline $1.5 \mathrm{~min}$ & $27 \%$ & $39 \%$ \\
\hline $6.5 \mathrm{~min}$ & $30 \%$ & $40 \%$ \\
\hline
\end{tabular}

It worth noticing that the results reported in Table 2 are measured while the 
hinge is held flat. Such constraint will slightly reduce the y-shrinkage, but this does not affect the discussion above considering the large differences between the $\mathrm{x}$ and y-shrinkage.

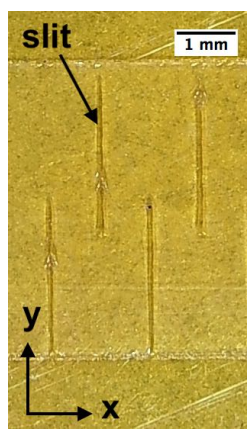

(a)

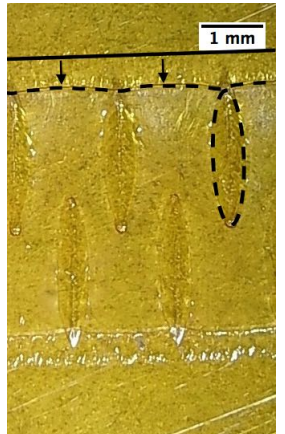

(b)

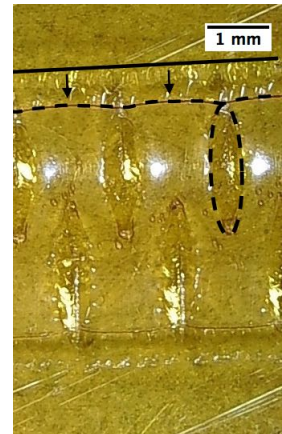

(c)

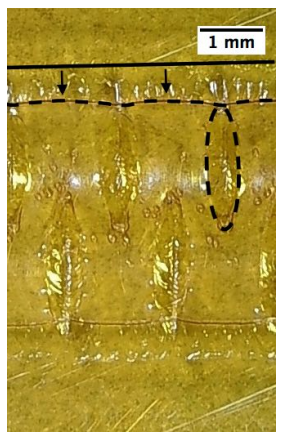

(d)

Figure 8: Optical microscopic images of the kirigami hinge (hinge lies along $\mathrm{x}$ direction): (a) before heating, (b) after $t=0.5 \mathrm{~min}$ of convective heating at $85^{\circ} \mathrm{C}$, (c) $t=1.5 \mathrm{~min}$ at $85^{\circ} \mathrm{C}$, (d) $t=6.5 \mathrm{~min}$ at $85^{\circ} \mathrm{C}$ (the dotted lines illustrate the expansion of the slits due to the Mylar shrinkage), note that the hinge is held flat while the images are taken.

\section{Experiment on a self-folding origami sheet}

This section demonstrates that the heat-activated kirigami hinge can be used to realise a new type of gossamer structure based on self-folding origami. Current gossamer structures are usually packed and stored as a folded origami, and then deploy and achieve the target shape under external supporting forces. However, the folded origami offers limited packing ratio especially for very large membranes, while keeping the membrane flat and rolling it onto a mandrel could realise the optimum packing. Therefore, we propose a gossamer structure that is stored as flat by winding into a cylinder and automatically fold along an origami crease pattern when unrolled and heated. This is illustrated by Figure 9 (not to scale), where the 2D film is stored on a mandrel, unrolled, and then folded. As mentioned in the introduction, this folded 3D configuration not only self-rigidises, but also has the potential to provide design-able properties and built-in functionalities of origami meta-structures. The 
origami reported in Figure 10 and 11 is based on an optical meta-structure solar sail reflector reported in a previous study [27]. It is designed to induce tuneable optical properties while achieving self-rigidisation. This origami uses a rigid-foldable non-flat degree-four vertex pattern with self-locking behaviour [43, 44, 45], which can be regarded as a variation of Miura-origami pattern [46, 47]. The detailed design is available from the previous paper [27].

Folding of an origami is naturally more predictable than a stand-alone hinge, since origami has limited degrees of freedom (the folding of origami creases are coupled). However, as shown by Figure 10, the methods preventing bifurcation are still necessary. The No. 1 membrane in the figure has hinges without slits, and the origami is barely folded due to the stress concentration in the Kapton regions where multiple hinges intersect. The No. 2 membrane is similar to No. 1, but holes are cut into the Kapton layer at the intersections of hinges to prevent stress concentration. It shows better folding behaviour, but bifurcation occurs in most of the hinges, leading to shape errors. The No. 3 and the No. 4 (a scaled-up version of No. 3) membranes use the design 2 kirigami hinges, and both fold as designed.

To prevent the tape-spring buckling as mentioned in section 2, all the samples shown in Figure 10 are heated while being constrained on a flat plate in order to guarantee the initial flatness of the membrane. The detailed activation process is described according to Figure 11. At the beginning (Figure 11a), the membrane is loosely bonded onto the flat plate at edges while leaving space for shrinkage. Then the first activation step is carried out by heating the membrane, together with the plate, in the oven at $85^{\circ} \mathrm{C}$ for $5 \mathrm{~min}$. The membrane is then removed from the oven (Figure 11b). It can be seen that all the hinges are curved, while no bifurcation or buckling are observed. Owing to the rigidising effect of the curved hinges, the membrane can be set free from the flat plate with no risk of further bifurcation or buckling. As shown by Figure 11c, the origami is cut off from the plate, and then reheated for another $5 \mathrm{~min}$. The final result (Figure 11d) shows that the origami is fully folded.

The reason why such out-of-plane constraint (from the flat plate) is necessary is discussed here. Since an ideal origami has only one degree of freedom, while a flexible membrane has infinite degrees of freedom due to its flexibility, a film with initial defect is likely to enter a state with a "wrong shape" if no external out-of-plane guiding force was applied [26, 48]. Automatic recovery to the target shape is hindered by a strain energy barrier between the "wrong shape" and the target shape [49, 50]. 


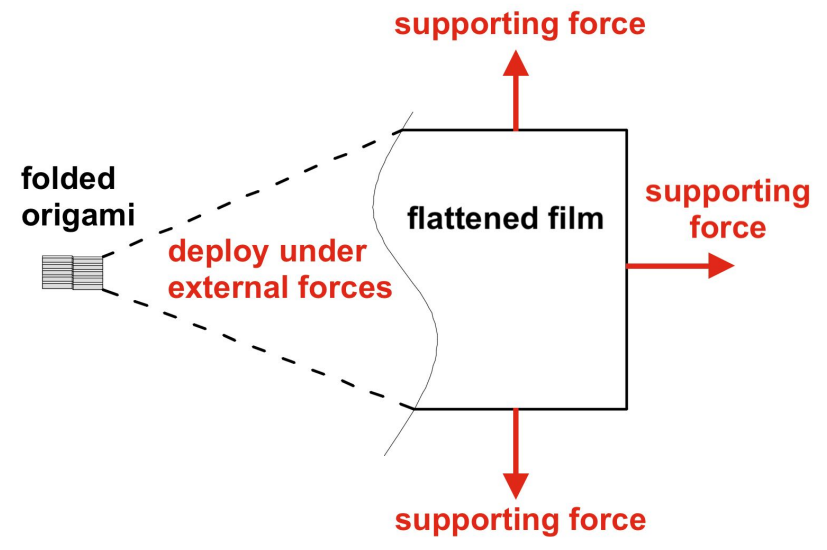

(a)

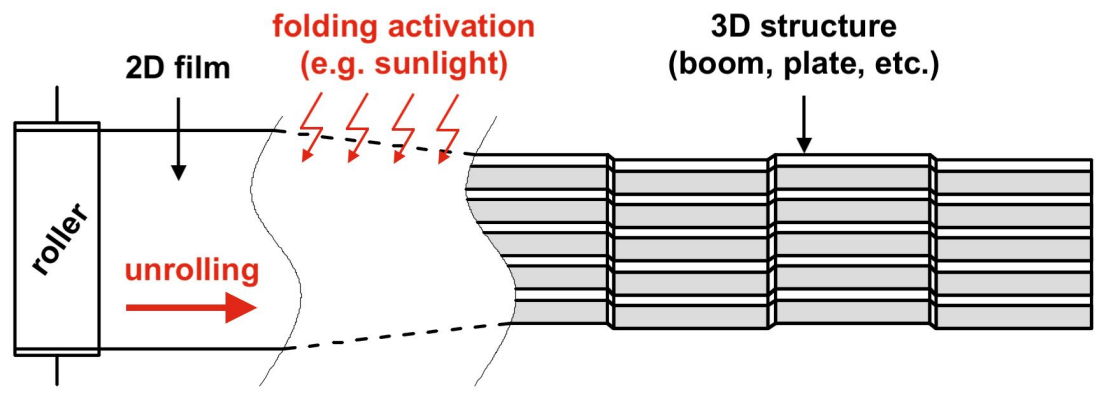

(b)

Figure 9: Unlike the conventional gossamer structures that are packed as folded origami and deploy by flattening under supporting forces (as illustrated in (a)), we propose a new solution (illustrated in (b)), where the gossamer structure is stored as flat by winding onto a roller, which maximises the volumetric packing ratio; then released by unrolling, and transformed into a 3D configuration by self-folding; the 3D structure can be designed to support load, and also provide built-in functionalities of origami meta-structures (sketches not to scale).

Although the hinge design with staggered slits (Figure 4d) has significantly improved the resistance to local buckling, it has limited contribution to the overall behaviour of a complex origami. However, by constraining the out-of-plane deformation (or in other words, the folding angle), the strain energy barrier between different shapes can be reduced [51], thus the target shape, which has the lowest strain energy, will finally 


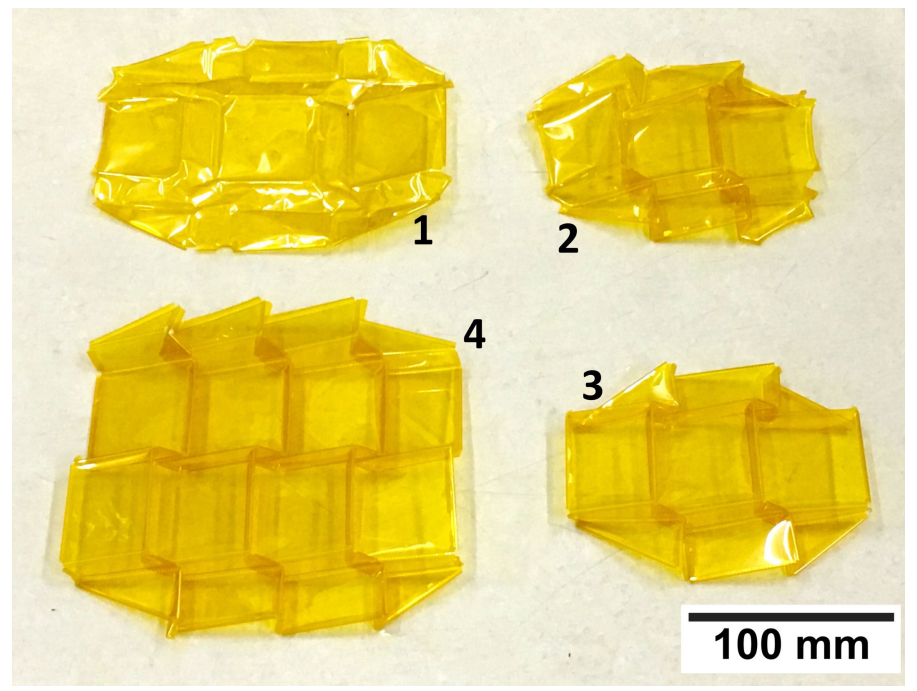

Figure 10: The self-folding membrane with a 4-vertex origami pattern, where 1: with no slits in the hinge, which barely folds along the origami pattern, 2: similar to 1 but with holes at the intersections of hinges to prevent stress concentration, which folds with significant shape error due to bifurcation at hinges, 3: similar to 2 but with kirigami hinges, which folds as designed, 4: a larger version of 3, which is also properly folded.

emerge. The speculative discussion above only describes the behaviour superficially, and more rigorous study is still required to understand such problem.

To summarise, by utilising the kirigami hinge design and keeping the membrane generally flat during the initial activation, bifurcation and buckling can be prevented. In real applications, parallel constraining plates, centrifugal force or mechanical tensioners can also be used to guarantee the flatness of membrane during the early stage of deployment and realise a flawless folding.

\section{Experiment on a self-forming tube}

Deployable tubular structures (booms) that can be rolled-up when flattened and then deploy to achieve high specific structural stiffness can be used as a universal structural element for deployable space structures [4, 8]. In this section, the kirigami hinge is used to realise a membrane structure that automatically transforms from a 


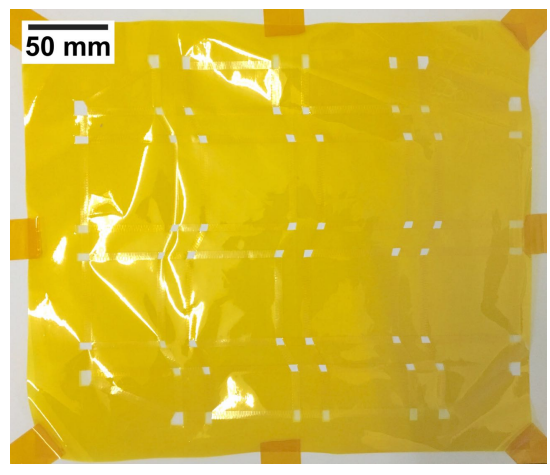

(a)

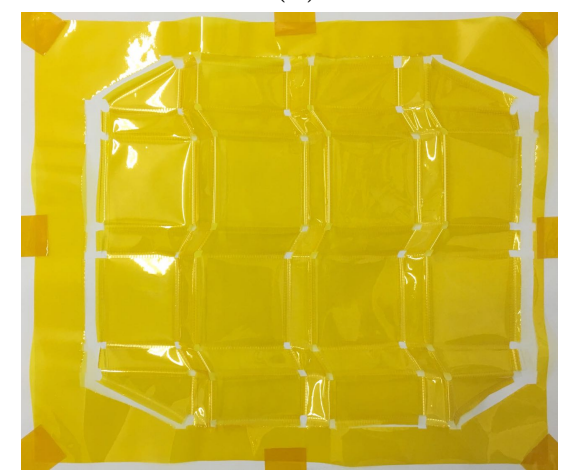

(c)

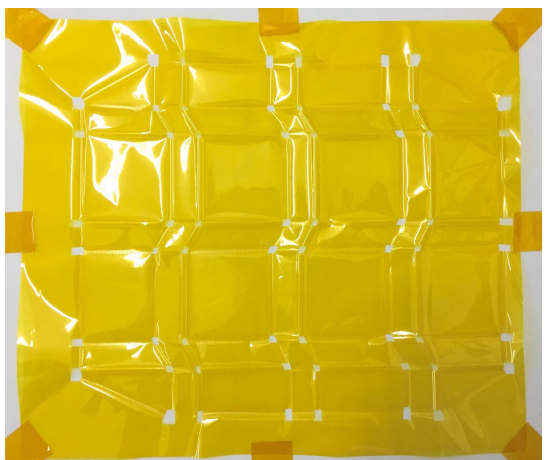

(b)

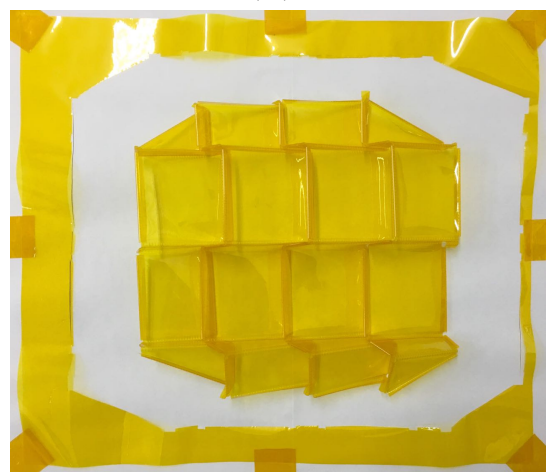

(d)

Figure 11: Process to activate the self-folding origami sheet: (a) the membrane is loosely bonded onto a flat plate to maintain flatness while leaving space for shrinkage during the initial activation step, (b) after the first hot-air heating of $5 \mathrm{~min}$ at $85^{\circ} \mathrm{C}$, the sheet shows no bifurcations, and the hinges are slightly curved, preventing them from tape-spring buckling, (c) the origami sheet is cut off from the plate before reheated, (d) the fully folded origami sheet after reheated at $85^{\circ} \mathrm{C}$ for $5 \mathrm{~min}$.

flat shape into a tube upon heating. This allows it to be embedded into a flexible membrane and then deploy to rigidise the gossamer structure. The design is shown in Figure 12, which consists of two kirigami hinges facing each other. When folded, each hinge will form one half of the tube that is bonded to the other half using the acrylic adhesive. In terms of thermal control, the feasibility of self-deployment upon exposure to sunlight is demonstrated in section 7 .

As shown by Figure 13 , the formation of the tube is completed in under $1.5 \mathrm{~min}$ 


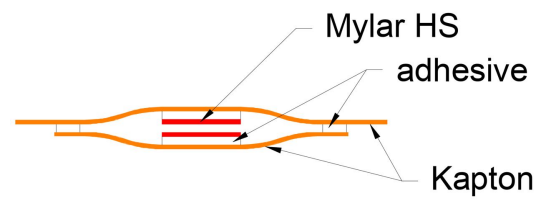

(a)

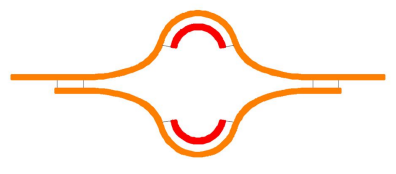

(b)

Figure 12: Cross-section of the self-forming tubular structure, (a) before heating, (b) after heating, the tube is formed with the kirigami hinge folded.

of heating at $85^{\circ} \mathrm{C}$, and the resulted structure shows a significantly higher stiffness in both bending and torsion. For instance, as shown in Figure 14, a cantilever tube made of $25 \mu \mathrm{m}$ thick Kapton film and $16 \mu \mathrm{m}$ Mylar that weighs $0.1 \mathrm{~g}$ and is $4 \mathrm{~cm}$ long can carry $4 g$ of load at its tip without any permanent deformation. As the load increases to $5 \mathrm{~g}$, local buckling is observed at the support end.

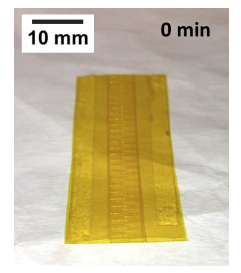

(a)

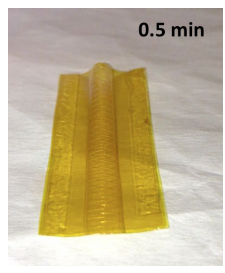

(b)

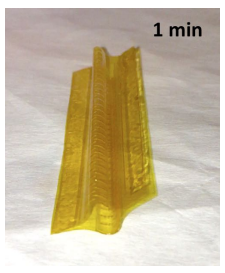

(c)

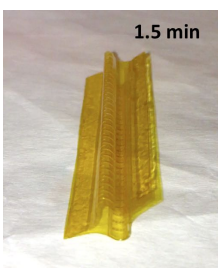

(d)

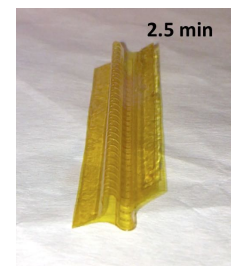

(e)

Figure 13: Formation of the tubular structure, (a) beginning of heating, (b) at $t=0.5 \mathrm{~min}$ at $85^{\circ} \mathrm{C}$, (c) $t=1 \mathrm{~min}$ at $85^{\circ} \mathrm{C}$, (d) $t=1.5 \mathrm{~min}$ at $85^{\circ} \mathrm{C}$ the formation is fully completed, (e) $t=2.5 \mathrm{~min}$ at $85^{\circ} \mathrm{C}$.

\section{Analysis of Sunlight-Activated Folding}

In space applications, a protective/reflective metallic layer is needed on the polymer membrane to protect it from environmental attack, improve the tear resistance, and increase photonic-propulsion when used on solar sails [52]. This section provides a proof-of-concept thermal analysis, which demonstrates the concept of utilising such metallic coatings to activate folding in orbital environment by absorbing solar energy.

The orbital thermal condition with the absence of convection is determined by radiation and conduction only. For a thin membrane facing the sun, the through- 


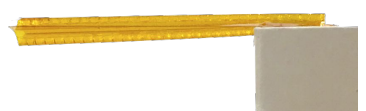

(a)

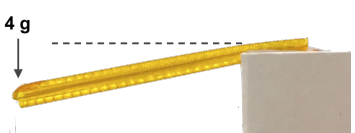

(b)

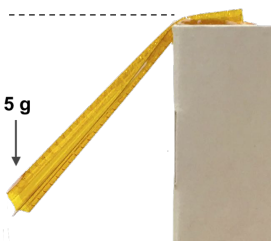

(c)

Figure 14: Bending of the cantilever self-forming tube of $4 \mathrm{~cm}$ length and $0.1 \mathrm{~g}$ structural mass, (a) initial shape without applied load, (b) the tube deflects under $4 \mathrm{~g}$ of load at its tip, (c) the tube buckles locally under a load of $5 \mathrm{~g}$.

thickness conduction is almost instant. Therefore, the temperature of a uniform film can be treated as homogeneous and determined by radiative heat transmission.

Conventional vapour-deposited metallic coatings can achieve various thermal radiation properties. For instance, an aluminium coating has a reflectivity close to 90\% throughout a wide spectrum including infrared, while a silver coating yields significantly above $90 \%$ [53]. In terms of an emissive coating that helps to radiate thermal energy away from a membrane for either cooling or heat transfer purposes, vapour-deposited chromium yields an emissivity of over $20 \%$. While for picking up heat from the environment, nickel provides a near $40 \%$ absorptivity and low emissivity [54]. Typical coating materials that can be useful for the thermal control of the proposed membrane are listed in Table 3.

Table 3: Typical coatings useful for the thermal control of the proposed self-folding membrane [54].

\begin{tabular}{c|c|c|c}
\hline Coating & Solar absorptivity & Emissivity & Typical usage \\
\hline Vapour-deposited Ag & $4 \%$ & $2 \%$ & Reflection \\
Vapour-deposited Al & $12 \%$ & $3 \%$ & Reflection \\
Vapour-deposited Ni & $38 \%$ & $4 \%$ & Absorption \\
Vapour-deposited Cr & $57 \%$ & $24 \%$ & Emission \& Heat dissipation \\
Carbon black paint & $96 \%$ & $88 \%$ & Absorption \& Emission \\
\hline
\end{tabular}

According to the discussion above, the temperature of a single membrane can be resolved using the equation below [53], which assumes thermal equilibrium between 
radiation absorption and emission:

$$
T=\sqrt[4]{\frac{\alpha \cdot q_{s}}{\sigma \cdot\left(\epsilon_{1}+\epsilon_{2}\right)}}
$$

Where $\epsilon_{1}$ and $\epsilon_{2}$ are the surface emissivities of the sun side and the rare side respectively. Consider a membrane directly facing the sun on a spacecraft in Low Earth Orbit (LEO), the solar radiation power density will be $q_{s}=1.36 \mathrm{~kW} / \mathrm{m}^{2}$ [53]. Here we provide several examples under this typical thermal control scenario. Assuming both sides of the membrane are vapour-deposited Al shown in Table 3 , and ignore the dependence of materials properties on temperature, the membrane will be at $195^{\circ} \mathrm{C}$. Changing the front coating to $\mathrm{Ag}$ will reduce the temperature to $99^{\circ} \mathrm{C}$. Such configurations can activate folding as the membrane temperature is well above the glass transition point of Mylar HS $\left(T_{g}=82{ }^{\circ} \mathrm{C}\right)$ to initiate shrinkage. In case a lower operating temperature is required, a vapour-deposited $\mathrm{Cr}$ coating can be used on the rear surface, which could reduce the membrane temperature of an $\mathrm{Al}$-coated membrane to $48^{\circ} \mathrm{C}$. Under such condition, the kirigami hinges need to be covered by light-absorbing materials on the sun side to activate folding. This can be achieved using the carbon black paint on top of the hinges, which leads to a hinge temperature of $106^{\circ} \mathrm{C}$.

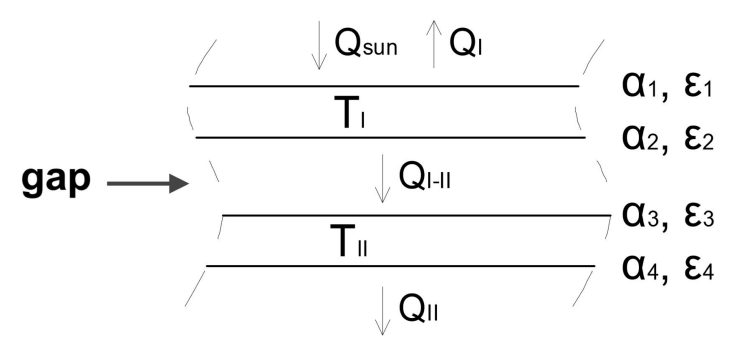

Figure 15: Thermal condition of two overlapped membranes with a gap in between.

Certain designs such as the self-forming tube discussed in section 6 (Figure 12. may use overlapped membranes with a gap (which is vacuum) in between, as shown schematically in Figure 15. Considering the thermal equilibrium of the whole structure, the absorbed solar energy equals the total radiative cooling, i.e.,

$$
Q_{\text {sun }}=Q_{I}+Q_{I I}
$$


Thus we have,

$$
\alpha \cdot W=\sigma \epsilon_{1} T_{I}^{4}+\sigma \epsilon_{4} T_{I I}^{4}
$$

The thermal equilibrium of the rear membrane alone, is given by:

$$
Q_{I-I I}=Q_{I I}
$$

According to literature [55], $Q_{I-I I}$ represents the radiative thermal transmission between two infinite planes and has the expression:

$$
Q_{I-I I}=\frac{\sigma \epsilon_{2} T_{I}{ }^{4} \cdot \alpha_{3}-\sigma \epsilon_{3} T_{I I}{ }^{4} \cdot \alpha_{2}}{\alpha_{2}+\alpha_{3}-\alpha_{2} \times \alpha_{3}}
$$

It should be noted that the radiation spectrum between the two membranes is very different to solar spectrum, and the absorptivities from Table 3 do not hold. For simplicity, assume the coatings on both sides of the gap in Figure 15 are identical, while the emissivity and absorptivity are the same for the surfaces with the same coating at similar temperatures: $\alpha_{2}=\alpha_{3}=\epsilon_{2}=\epsilon_{3}=\alpha=\epsilon$, an Ag front coating and an $\mathrm{Al}$ back coating are used. Then the membranes temperature $T_{I}$ and $T_{I I}$ can be evaluated using Eq. 225. The result (Figure 16) suggests that self-folding upon exposure to sunlight, which requires $T_{I}>82^{\circ} \mathrm{C}$ and $T_{I I}>82^{\circ} \mathrm{C}$, can be achieved with a wide range of absorptivity and emissivity $(\alpha, \epsilon>0.1)$.

Furthermore, the vapour-deposited metallic coating changes the flexural rigidity of the polymer membranes and thus the resistance to folding. Consider a Kapton film with $100 \mathrm{~nm} \mathrm{Al}$ coating on both sides, then assuming $70 \mathrm{GPa}$ and $2.5 \mathrm{GPa}$ Young's modulus for $\mathrm{Al}$ and Kapton, respectively, the flexural rigidity, $D$ of the coated and uncoated film can be evaluated according to Euler-Bernoulli linear beam theory, where $I$ is the second moment of area (geometric parameter) [56]:

$$
\begin{aligned}
& D_{\text {coated }}=E_{A l} I_{A l_{1}}+E_{\text {kapton }} I_{\text {kapton }}+E_{A l} I_{A l_{2}} \\
& D_{\text {uncoated }}=E_{\text {kapton }} I_{\text {kapton }}
\end{aligned}
$$

The ratio between the flexural rigidity, $D_{\text {coated }} / D_{\text {uncoated }}$, is then plotted in Figure 17. It can be seen that the effect of coating on the membrane's bending rigidity increases with decreasing Kapton thickness. The rigidity ratio is 1.47 for the $25 \mu \mathrm{m}$ Kapton used in the present study, and such coating will have no significant effect on the self-folding behaviour of the proposed polymer membrane. 


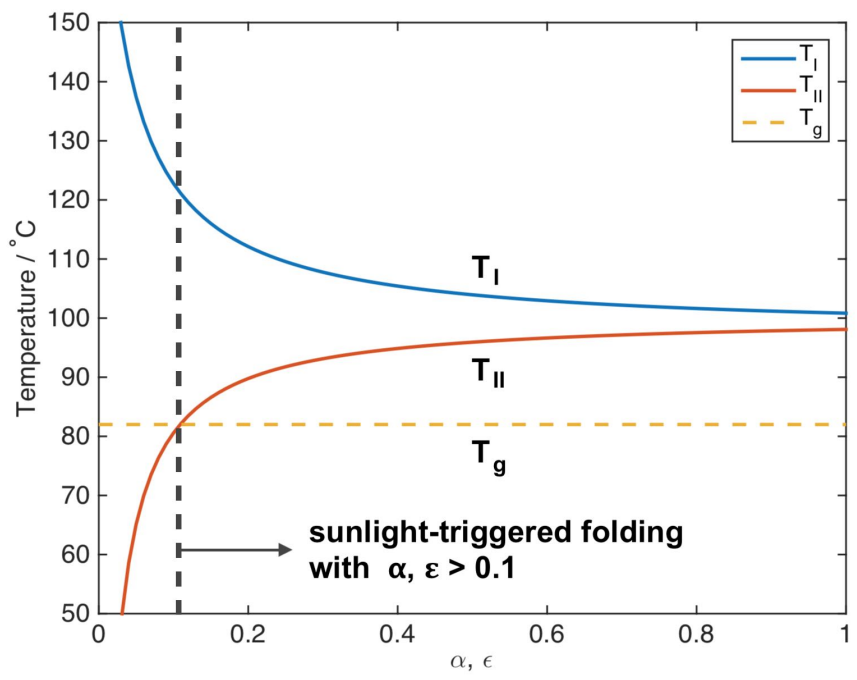

Figure 16: Temperature of two overlapped membranes, $T_{I}$ and $T_{I I}$ plotted against the absorptivity $\alpha$ and emissivity $\epsilon$ of the coatings on both sides of the gap between the membranes, showing that the glass transition point of Mylar HS, $T_{g}=82^{\circ} \mathrm{C}$ can be reached with $\alpha$ and $\epsilon>0.1$.

\section{Summary}

Self-folding membrane that can permanently fold along complex crease patterns when heated up to a certain temperature is designed and fabricated. It is based on a thermally stable Kapton substrate, and folding is activated by an isotropic heat shrinking film, Mylar HS, which is bonded along the hinges using a flexible acrylic adhesive. The specially designed kirigami hinges have slits cut into the Mylar and the adhesive layers to relax the stress caused by shrinkage and convert the isotropic shrinkage to unidirectional (i.e., anisotropic), thereby realise a predictable folding without bifurcation. In comparison with the existing self-folding membranes, the proposed innovative design is based on space-qualified materials, and is potentially mass-producible. It also features weight saving since most part of the membrane is single layered Kapton and only the hinge region is multi-layered, which is important for certain space missions such as solar sails.

Test samples of a self-folding origami structure and a self-forming tubular structure are fabricated using a semi-automated production method that can 


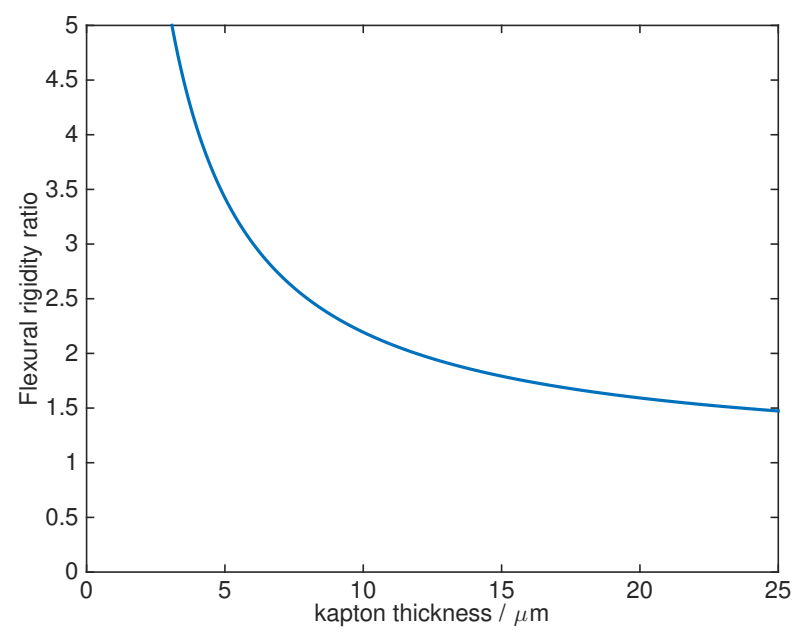

Figure 17: Ratio between the flexural rigidity of coated and uncoated membranes, $D_{\text {coated }} / D_{\text {uncoated }}$, plotted against Kapton thickness, assuming aluminium coatings with $100 \mathrm{~nm}$ thickness on both sides of the Kapton film.

potentially be converted to an industrial roll-to-roll process. Tests carried out in an oven suggest that the kirigami hinge design, as well as the actuation process that keeps the membrane generally flat during the deployment has successfully prevented bifurcation. The 2D membranes were successfully transformed into 3D structures.

Furthermore, thermal radiation analysis has demonstrated that the folding can be activated using sunlight in a space environment by applying conventional spacecraft thermal control coatings onto the membranes. This creates a different type of deployable gossamer structures: when stored, the flexible membrane can be winded up into a cylinder instead of packed as a pre-folded origami, which potentially saves stowage volume and allows simpler packaging process; for deployment, the membrane is released from the package and heated by sunlight, which activates the folding along a pre-defined crease pattern. The folded membrane not only achieves self-rigidisation, but could also serve as a building block of future origami metastructures with various design-able behaviours and built-in functionalities. 


\section{Reference}

[1] Jenkins CH. Progress in Astronautics and Aeronautics: Gossamer Spacecraft: Membrane and Inflatable Structures Technology for Space Applications. vol. 191. Aiaa; 2001.

[2] Ruggiero EJ, Inman DJ. Gossamer spacecraft: recent trends in design, analysis, experimentation, and control. Journal of Spacecraft and Rockets. 2006;43(1):10-24.

[3] Fu B, Sperber E, Eke F. Solar sail technologyA state of the art review. Progress in Aerospace Sciences. 2016;86:1-19.

[4] Block J, Straubel M, Wiedemann M. Ultralight deployable booms for solar sails and other large gossamer structures in space. Acta Astronautica. 2011;68(7):984-992.

[5] Roberts PC, Harkness PG. Drag sail for end-of-life disposal from low earth orbit. Journal of Spacecraft and Rockets. 2007;44(6):1195-1203.

[6] Santiago-Prowald J, Baier H. Advances in deployable structures and surfaces for large apertures in space. CEAS Space Journal. 2013;5(3-4):89-115.

[7] Chu Z, Deng Z, Qi X, Li B. Modeling and analysis of a large deployable antenna structure. Acta Astronautica. 2014;95:51-60.

[8] Puig L, Barton A, Rando N. A review on large deployable structures for astrophysics missions. Acta Astronautica. 2010;67(1):12-26.

[9] Garner C, Diedrich B, Leipold M. A Summary of Solar Sail Technology Developments and Proposed Demonstration Missions. In: AIAA-99-2697, presented at 35th AIAA Joint Propulsion Conference. Citeseer; 1999. .

[10] Häuplik-Meusburger S, Sommer B, Aguzzi M. Inflatable technologies: adaptability from dream to reality. Acta Astronautica. 2009;65(5):841-852.

[11] Babuscia A, Corbin B, Knapp M, Jensen-Clem R, Van de Loo M, Seager S. Inflatable antenna for cubesats: Motivation for development and antenna design. Acta Astronautica. 2013;91:322-332.

[12] Hedgepeth JM. Dynamics of a large spin-stiffened deployable paraboloidal antenna. Journal of Spacecraft and Rockets. 1970;7(9):1043-1048.

[13] MacNeal RH. The heliogyro - an interplanetary flying machine. NASA Contractors Report CR 84460, 1967;

[14] Janhunen P. Photonic spin control for solar wind electric sail. Acta Astronautica. 2013;83:8590.

[15] Mao H, Sinn T, Vasile M, Tibert G. Post-launch analysis of the deployment dynamics of a space web sounding rocket experiment. Acta Astronautica. 2016;127:345-358.

[16] Gardsback M, Tibert G. Deployment control of spinning space webs. Journal of guidance, control, and dynamics. 2009;32(1):40.

[17] Vinogradov AM, Jenkins CH, Su J, Bar-Cohen Y, Pollard EL. Functional Materials for Smart Gossamer Spacecraft. Progress in Astronautics and Aeronautics. 2006;212:165.

[18] Salama M, Jenkins C. Intelligent gossamer structures-A review of recent developments and future trends. In: 19th AIAA Applied Aerodynamics Conference; 2013. p. 1196.

[19] Cadogan DP, Scarborough SE. Rigidizable materials for use in gossamer space inflatable structures. AIAA paper. 2001;1417:2001. 
[20] Bryant RG, Seaman ST, Wilkie WK, Miyauchi M, Working DC. Selection and manufacturing of membrane materials for solar sails. In: Advances in Solar Sailing. Springer; 2014. p. $525-540$.

[21] Blomquist RS. Heliogyro control. Ph.D. Thesis, Carnegie Mellon University, Pittsburg, Pennsylvania; 2009.

[22] Liu Y, Genzer J, Dickey MD. "2D or not 2D": Shape-programming polymer sheets. Progress in Polymer Science. 2016;52:79-106.

[23] Dudte LH, Vouga E, Tachi T, Mahadevan L. Programming curvature using origami tessellations. Nature materials. 2016;15(5):583-588.

[24] Lv C, Krishnaraju D, Konjevod G, Yu H, Jiang H. Origami based mechanical metamaterials. Scientific reports. 2014;4:5979-5981.

[25] Filipov ET, Tachi T, Paulino GH. Origami tubes assembled into stiff, yet reconfigurable structures and metamaterials. Proceedings of the National Academy of Sciences. 2015;112(40):12321-12326.

[26] Liu X, Gattas JM, Chen Y. One-DOF Superimposed Rigid Origami with Multiple States. Scientific reports. 2016;6:36883.

[27] Wu R, Roberts PC, Soutis C, Diver C. Heliogyro Solar Sail with Self-Regulated Centrifugal Deployment Enabled by an Origami-Inspired Morphing Reflector. Acta Astronautica. 2018;Accepted manuscript.

[28] Deng D, Yang Y, Chen Y, Lan X, Tice J. Accurately controlled sequential self-folding structures by polystyrene film. Smart Materials and Structures. 2017;26(8):085040.

[29] Tolley MT, Felton SM, Miyashita S, Aukes D, Rus D, Wood RJ. Self-folding origami: shape memory composites activated by uniform heating. Smart Materials and Structures. 2014;23(9):094006.

[30] Miyashita S, Meeker L, Tolley MT, Wood RJ, Rus D. Self-folding miniature elastic electric devices. Smart Materials and Structures. 2014;23(9):094005.

[31] Cui J, Adams J, Zhu Y. Pop-up assembly of 3D structures actuated by heat shrinkable polymers. Smart Materials and Structures. 2017;26(12):125011.

[32] Abdullah AM, Braun PV, Hsia KJ. Bifurcation of self-folded polygonal bilayers. Applied Physics Letters. 2017;111(10):104101.

[33] Caruso N, Cvetkovi A, Lucantonio A, Noselli G, Desimone A. Spontaneous morphing of equibiaxially pre-stretched elastic bilayers: the role of sample geometry. International Journal of Mechanical Sciences, 2017;

[34] Liu Y, Miskiewicz M, Escuti MJ, Genzer J, Dickey MD. Three-dimensional folding of pre-strained polymer sheets via absorption of laser light. Journal of Applied Physics. 2014;115(20):043505-12445.

[35] Lee Y, Lee H, Hwang T, Lee JG, Cho M. Sequential Folding using Light-activated Polystyrene Sheet. Scientific Reports. 2015;5:16544.

[36] Davis D, Mailen R, Genzer J, Dickey MD. Self-folding of polymer sheets using microwaves and graphene ink. Rsc Advances. 2015;5(108):89254-89261.

[37] Ryu J, DAmato M, Cui X, Long KN, Qi HJ, Dunn ML. Photo-origamiBending and folding polymers with light. Applied Physics Letters. 2012;100(16):82-87. 
[38] Leong TG, Benson BR, Call EK, Gracias DH. Thin Film Stress Driven Self-Folding of Microstructured Containers. Small. 2008;4(10):16051609.

[39] Bassik N, Stern GM, Gracias DH. Microassembly based on hands free origami with bidirectional curvature. Applied Physics Letters. 2009;95(9):91901.

[40] Leong TG, Randall CL, Benson BR, Bassik N, Stern GM, Gracias DH. Tetherless thermobiochemically actuated microgrippers. Proceedings of the National Academy of Sciences of the United States of America. 2009;106(3):703.

[41] Na JH, Evans AA, Bae J, Chiappelli MC, Santangelo CD, Lang RJ, et al. Programming reversibly self-folding origami with micropatterned photo-crosslinkable polymer trilayers. Advanced Materials. 2015;27(1):79-85.

[42] Seffen K, Pellegrino S. Deployment dynamics of tape springs. In: Proceedings of the Royal Society of London A: Mathematical, Physical and Engineering Sciences. vol. 455. The Royal Society; 1999. p. 1003-1048.

[43] Fang H, Li S, Wang K. Self-locking degree-4 vertex origami structures. Proc R Soc A. 2016;472(2195):20160682.

[44] Fang H, Li S, Xu J, Wang K. Locking mechanisms in degree-4 vertex origami structures. In: Active and Passive Smart Structures and Integrated Systems 2016. vol. 9799. International Society for Optics and Photonics; 2016. p. 979910.

[45] Gattas JM, Wu W, You Z. Miura-base rigid origami: parameterizations of first-level derivative and piecewise geometries. Journal of Mechanical Design. 2013;135(11):111011.

[46] Sareh P, Guest SD. A Framework for the Symmetric Generalisation of the Miura-ori. International Journal of Space Structures. 2015;30(2):141-152.

[47] Sareh P, Guest SD. Design of non-isomorphic symmetric descendants of the Miura-ori. Smart Materials and Structures. 2015;24(8):085002.

[48] Santangelo CD. Extreme Mechanics: Self-Folding Origami. Annual Review of Condensed Matter Physics. 2017;8:165-183.

[49] Silverberg JL, Evans AA, McLeod L, Hayward RC, Hull T, Santangelo CD, et al. Using origami design principles to fold reprogrammable mechanical metamaterials. Science. 2014;345(6197):647-650.

[50] Silverberg JL, Na JH, Evans AA, Liu B, Hull TC, Santangelo CD, et al. Origami structures with a critical transition to bistability arising from hidden degrees of freedom. Nature materials. 2015;14(4):389.

[51] Liu B, Silverberg JL, Evans AA, Santangelo CD, Lang RJ, Hull TC, et al. Topological kinematics of origami metamaterials. Nature Physics. 2018;p. 1.

[52] Macdonald M. Advances in solar sailing. Springer Praxis, Chichester, UK; 2014.

[53] Wright JL. Space sailing. Gordon and Breach Science Publications, Amsterdam; 1992.

[54] Kauder L. Spacecraft thermal control coatings references. NASA/TP-2005-212792, 2005;.

[55] Spakovszky Z. 16. Unified: Thermodynamics and Propulsion;. Unified Engineering, 2009. http://web.mit.edu/16.unified/www/SPRING/propulsion/notes/notes.html, (accessed 1 Dec 2017).

[56] Gere JM, Goodno BJ. Mechanics of materials, 8th. Stamford, Connecticut: Cengage Learning; 2012 . 